\title{
Dienogest in long-term treatment of endometriosis
}

This article was published in the following Dove Press journal:

International Journal of Women's Health

5 July 2011

Number of times this article has been viewed

\section{Adolf E Schindler \\ Institute for Medical Research and Education, Essen, Germany}

Correspondence: Adolf Schindler Institut für Medizinische Forschung und Fortbildung, Universitätsklinikum, Hufelandstrasse 55 D-45I 22 Essen,

Germany

Tel +492017991833

Fax +4920I 7499533

Email adolf.schindler@uni-due.de
Abstract: Endometriosis is a chronic disease primarily affecting women of childbearing age, in which endometriotic lesions form outside the uterus, typically leading to painful symptoms, fatigue, and infertility. The symptoms of endometriosis may cause significant impairment in quality of life and represent a substantial economic burden to patients, families, and society. There is no cure for endometriosis; management consists of alleviating pain and other symptoms, reducing endometriotic lesions, and improving quality of life. Recurrence after surgical intervention is common, while the clinical evidence to support the efficacy and safety of many medications currently used in endometriosis is limited. Dienogest is an oral progestin that has been investigated extensively in the treatment of endometriosis in two clinical programs performed in Europe and Japan, including dose-ranging, placebo-controlled, active comparator-controlled, and long-term (up to 65 weeks) studies. These studies demonstrated that dienogest $2 \mathrm{mg}$ daily effectively alleviates the painful symptoms of endometriosis, reduces endometriotic lesions, and improves indices of quality of life. Dienogest showed a favorable safety and tolerability profile in these studies, with predictable adverse effects, high rates of patient compliance, and low withdrawal rates. This review article describes the clinical trial evidence that characterizes the efficacy and safety of dienogest in endometriosis, including two studies characterizing dienogest in long-term use. The relevance of these findings to the management of endometriosis in clinical practice is discussed.

Keywords: dienogest, endometriosis, progestins, long-term treatment, quality of life, symptoms, pain

\section{Endometriosis: a chronic, painful disease}

Endometriosis is a chronic, estrogen-dependent disease that affects approximately $10 \%$ of women of reproductive age, with a peak incidence in the age range of 25-30 years. ${ }^{1-3}$ Endometriosis is characterized by the formation of endometriotic lesions outside the uterus, including the ovaries and other pelvic structures. These lesions cause a chronic, inflammatory reaction, which can lead to the formation of scar tissue and adhesions. ${ }^{4}$ Women with endometriosis frequently experience symptoms of dysmenorrhea, premenstrual pain, dyspareunia, and chronic fatigue. ${ }^{5}$ Endometriosis can also interfere with functioning of the bowel or bladder, depending on the site where endometriotic lesions develop. Up to $50 \%$ of women with endometriosis experience infertility. ${ }^{6}$ However, the clinical presentations of endometriosis can vary widely, and many affected women are asymptomatic. ${ }^{7}$ No clear relationship exists between the extent of endometriotic lesions and a woman's symptoms.

Quality of life studies show that symptoms of endometriosis impact on many aspects of a woman's life, including work and education, relationships, and social functioning. ${ }^{8-14}$ 
As symptoms become more severe, quality of life is reduced further. In a recent international survey, women with endometriosis reported a substantial 38\% reduction in work productivity, which was attributable primarily to reduced work effectiveness in the presence of pelvic pain. ${ }^{15}$ Endometriosis also impacts mental health, with one study showing that $87 \%$ of the women investigated with endometriosis had depressive symptoms and $88 \%$ had anxiety. ${ }^{16}$ The severity of anxiety symptoms correlated with the intensity of pain.

Endometriosis places a considerable economic burden on families and on society. Delays in diagnosis, high rates of hospital admission, surgical procedures, and incidences of comorbid conditions contribute to make endometriosis a more costly public health problem than other chronic conditions such as migraine and Crohn's disease..$^{9,17-21}$

\section{Diagnosis and treatment of endometriosis Diagnosis}

Due to the variable presentation of endometriosis, there is typically a delay between the first appearance of symptoms and an accurate diagnosis. An international survey reported that this delay is, on average, seven years. ${ }^{15}$ Early diagnosis is an important objective, as endometriosis typically follows a progressive course characterized by a worsening of symptoms in the absence of effective treatment. Errors in diagnosis, with the potential for inappropriate therapy, are also common, creating anxiety and frustration, and contributing further to the burden of endometriosis.

A definitive diagnosis of endometriosis requires laparoscopy, ideally combined with confirmatory histology, to characterize endometriotic lesions. ${ }^{4,22}$ In practice, however, this invasive approach is considered unnecessary or inappropriate for many patients, and a presumptive diagnosis of endometriosis can be made from the symptoms alone. ${ }^{4}$

\section{Treatment}

There is no permanent cure for endometriosis. As stated by the American Society for Reproductive Medicine, "Endometriosis should be viewed as a chronic disease that requires a life-long management plan with the goal of maximizing the use of medical treatment and avoiding repeated surgical procedures." ${ }^{23}$ Women with endometriosis require ongoing, collaborative, supportive management of their condition, as well as an understanding of the significant impact that the condition can have on their quality of life.

The main aims of treatment are to alleviate pain and other symptoms, reduce endometriotic lesions, and improve the quality of life of affected individuals. A number of medical and surgical therapies are available to treat endometriosis, which may, on occasion, be used in combination. No single treatment is ideal for all patients and the management approach chosen should be directed to the individual needs of each patient. As endometriosis is a chronic disease, consideration should be given not only to the efficacy but also to the long-term safety and tolerability of the treatment options that are available.

\section{Surgery}

Surgical intervention includes ablation of endometriotic lesions, removal of endometriotic cysts, and division of adhesions. Surgery can provide pain relief and enhance fertility, but this approach should be delayed for as long as possible due to the high risk of recurrence, which attains a rate of $40 \%-50 \%$ at five years. ${ }^{24}$ Medications administered postoperatively may reduce the risk of recurrence.

\section{Medical therapy}

Nonsteroidal anti-inflammatory drugs are frequently used by women with endometriosis in an attempt to achieve analgesia, although clinical trial evidence to support the efficacy of these agents in endometriosis is lacking. ${ }^{25}$ A major limitation to the long-term use of nonsteroidal anti-inflammatory drugs is their significant side effects, including the risk of gastric ulceration and an antiovulatory effect when taken at mid-cycle. ${ }^{4,25}$

Specific medical therapies that are approved for the treatment of endometriosis include gonadotropin-releasing hormone ( $\mathrm{GnRH})$ agonists, danazol, and certain progestins. These agents and the combined oral contraceptives (COCs) share a common hormonal mechanism of action in endometriosis.

COCs are widely used to treat the symptoms of endometriosis, although they are not approved for this indication in the majority of countries because of the absence of supportive trial evidence. ${ }^{26}$ For the same reason, practice guidelines can offer limited guidance on the optimal COC regimens in endometriosis. ${ }^{27} \mathrm{~A}$ recently published review notes that the putative biological effects for COCs include both inhibition of endometrial cell implantation but also a protective effect against endometrial lesion necrosis. ${ }^{28}$ Clinical experience indicates that COCs may be used with safety in many women for the long-term treatment of endometriosis. However, a common problem with long-term continuous COC regimens is breakthrough bleeding. This is often treated by discontinuing the COC for a few days and then restarting therapy. 
GnRH agonists are an established therapy for endometriosis that can be administered via either intramuscular, subcutaneous, or intranasal routes. ${ }^{29}$ Depot formulations are available. Although GnRH agonists provide effective pain relief and reduce the progression of endometriotic implants, ${ }^{29}$ the hypoestrogenic state that they induce is associated with effects such as accelerated bone mineral density loss, hot flushes, and vaginal dryness. ${ }^{30}$ In consequence, the use of GnRH agonists is limited to six months in the absence of "add-back" therapy with steroids. The optimal regimens for add-back therapy are not yet established. ${ }^{18,31}$ For younger women who have not yet reached maximum bone density, guidelines recommend a careful consideration of the use of $\mathrm{GnRH}$ agonists due to their bone demineralizing effects. ${ }^{4}$

Danazol is an androgenic steroid that is effective in treating the signs and symptoms of endometriosis, but its use is limited by adverse effects on lipid metabolism and by weight gain, edema, acne, vaginal dryness, hot flushes, oily skin, hirsutism, liver toxicity, and breast atrophy. ${ }^{18,32}$ Because of these effects, danazol has today been superseded in many countries by alternative agents.

Oral, parenteral, intrauterine, or implantable progestins have been used for decades in the treatment of endometriosis, although for many of these agents there is a lack of supportive evidence from controlled clinical trials. Dose-finding data are lacking for most progestins, and there are few comparative data to indicate the benefits of one progestin over another.

The progestins that are approved for use in endometriosis vary between countries. A notable example is medroxyprogesterone acetate injectable suspension (Depo-subQ Provera $104^{\mathrm{TM}}$, Pfizer, New York, NY), which was approved recently by the US Food and Drug Administration for the treatment of endometriosis based on active comparator-controlled trial data. This medication carries a black box warning concerning possible bone mineral loss. The preparation is not available in Europe.

\section{Dienogest}

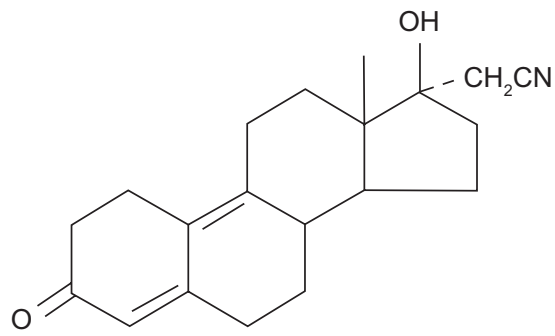

When administered continuously, progestins are effective in many women for the management of pain and other symptoms of endometriosis, with beneficial effects also relating to amenorrhea and anovulation. However, certain progestins are effective in endometriosis only at high doses when compared with use in other indications, ${ }^{33,34}$ which may increase the likelihood of adverse effects, such as weight gain and androgenic effects, and elevate the risk of cardiovascular adverse events.

\section{Dienogest in endometriosis}

Dienogest is an oral progestin that has been investigated systematically for the treatment of endometriosis in dose-ranging, placebo-controlled, active comparator-controlled, and long-term trials performed in Europe and Japan (Figure 1). Based on this trial evidence, dienogest has received approval as a monotherapy for the treatment of endometriosis in Europe, Japan, Australia, and Singapore.

\section{Preclinical studies}

Dienogest demonstrates a number of characteristics in preclinical studies that are relevant to its use in endometriosis. Pharmacologically, dienogest combines the advantages of the 19-norprogestin and the progesterone derivative classes. ${ }^{35,36}$ Dienogest binds to the progesterone receptor with high specificity, and produces a potent progestogenic effect related to the high circulating levels of the unbound molecule. ${ }^{37}$ Binding affinities of dienogest for estrogen, glucocorticoid, and mineralocorticoid receptors are negligible. Unlike other agents in the 19-norprogestin class, dienogest lacks androgenic effects; rather, dienogest has beneficial antiandrogenic properties typical of the progesterone derivatives, which are associated with minimal changes in lipid and carbohydrate levels. ${ }^{38,39}$

Dienogest is almost completely absorbed, and has a high bioavailability after oral administration, similar to other 19-norprogestins. Its relatively short half-life of 10 hours means there is no risk of accumulation after repeated dosing.

\section{Progesterone}

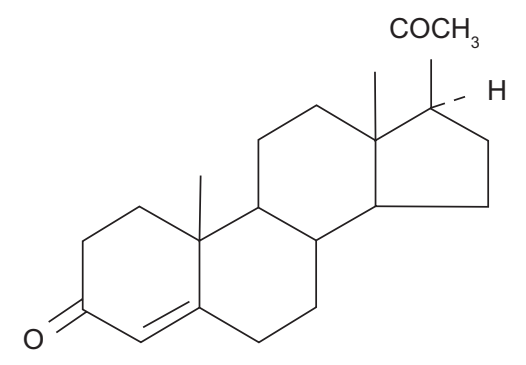

Figure I Structural formula of the progestin, dienogest, compared with naturally occurring progesterone. 
The majority of orally administered dienogest is excreted within 24 hours, mainly in urine.

Dienogest reduces endometriotic lesions through a number of biological mechanisms. Dienogest is associated with relatively moderate inhibition of gonadotropin secretion, leading to a modest reduction in the endogenous production of estradiol. ${ }^{40}$ When given continuously, dienogest induces a hypoestrogenic, hypergestagenic local endocrine environment, causing a decidualization of endometrial tissue followed by atrophy of the endometriotic lesions. Animal studies indicate that dienogest may also reduce plasma estradiol levels directly, through inducing apoptosis of granulosa cells in the ovary. ${ }^{41}$

A recent pharmacokinetic study confirmed the moderate suppression of estradiol levels, remaining within the lower end of the normal physiological range, in women volunteers administered dienogest at doses of $0.5-3 \mathrm{mg}$ daily. ${ }^{42}$ In this study, ovarian activity was effectively suppressed by the $2 \mathrm{mg}$ and $3 \mathrm{mg}$ doses, with a rapid return to ovulation after cessation of dienogest administration.

In exploratory models of endometriosis, dienogest also demonstrates antiproliferative, anti-inflammatory, and antiangiogenic effects. ${ }^{36,43-45}$ In vitro and animal studies show that dienogest has a direct inhibitory effect on the proliferation of endometrial-like tissue that is independent of actions via the progesterone receptor. ${ }^{46-49}$ Indirect antiinflammatory activity through modification of proinflammatory markers has been demonstrated for dienogest in in vitro and in vivo experiments. ${ }^{50} \mathrm{~A}$ further potential mechanism of action for dienogest is inhibition of angiogenesis, which represents an essential stage in the development of endometriotic lesions. Oral administration of dienogest significantly suppressed angiogenesis in a mouse model. ${ }^{45}$ The molecular mechanisms underlying these effects continue to be explored. . $^{48-51}$

\section{Clinical studies}

Clinical studies of dienogest with durations between 12 and 24 weeks have provided information on optimal dosing and on efficacy and safety characteristics that are relevant to the long-term management of endometriosis.

The optimal daily dose of dienogest for treating endometriosis was investigated in an open-label, randomized, multicenter, 24-week, dose-ranging study in Europe. ${ }^{39}$ Sixty-eight women with endometriosis stage I-III at laparoscopy (ie, with minimal to moderate disease) were randomly assigned to treatment with dienogest 1, 2, or $4 \mathrm{mg}$ once daily. Randomization to the $1 \mathrm{mg}$ group was halted prematurely because of unsatisfactory bleeding patterns. Laparoscopy showed that dienogest at $2 \mathrm{mg}$ and $4 \mathrm{mg}$ daily significantly reduced endometriotic lesions. In addition, dienogest at both these doses improved patient-reported symptoms, including the intensity of dyspareunia, dysmenorrhea, and diffuse pelvic pain. The $2 \mathrm{mg}$ and $4 \mathrm{mg}$ dienogest doses were generally well tolerated and rates of discontinuations due to adverse events were low. Irregular uterine bleeding was experienced by $55.2 \%$ in the $2 \mathrm{mg}$ group and $68.6 \%$ in the $4 \mathrm{mg}$ group, with a trend to decreased intensity over time in both groups. Based on these outcomes, dienogest at $2 \mathrm{mg}$ once daily was recommended as the optimal dose in the treatment of endometriosis.

In support of these findings, a Japanese study investigated dienogest at daily doses of $1 \mathrm{mg}, 2 \mathrm{mg}$, and $4 \mathrm{mg}$ for 24 weeks in 183 women with endometriosis. ${ }^{52}$ The $2 \mathrm{mg}$ and $4 \mathrm{mg}$ daily doses were equivalent in efficacy, measured as a global improvement in subjective symptoms. While safety assessments indicated no dose-related differences, reductions in estradiol levels associated with the $4 \mathrm{mg}$ dose indicated that $2 \mathrm{mg}$ daily may offer least potential for adverse effects on bone mineral density. Mean serum estradiol concentrations at between eight weeks and the end of treatment in this study were $84.5,37.4$, and $26.2 \mathrm{pg} / \mathrm{mL}$ for dienogest doses of 1,2 , and $4 \mathrm{mg} /$ day, respectively. A serum estradiol concentration in the range of $30-50 \mathrm{pg} / \mathrm{mL}$ is considered to fulfill the requirements of the estrogen threshold hypothesis, ${ }^{53}$ by which estrogen levels are suppressed sufficiently to inhibit endometriotic lesion growth, but are adequate to prevent hypoestrogenic side effects such as bone mineral loss. Of relevance to long-term compliance, $77 \%$ of the patients commented that they "wanted" or "definitely wanted" to use the dienogest $2 \mathrm{mg}$ daily dose again.

The effectiveness of dienogest at the $2 \mathrm{mg}$ daily dose was compared against placebo in a 12-week, randomized trial using a range of tools for measuring changes in symptoms and quality of life. The study enrolled 198 women with stage I-IV (ie, minimal to severe) endometriosis and an endometriosis-associated pelvic pain score of at least $30 \mathrm{~mm}$ on a visual analog scale (measured on a scale of $0-100 \mathrm{~mm}$ ), which represents a well validated tool for the measurement of pain. ${ }^{54,55}$ Dienogest produced a statistically significant decrease in the mean visual analog score compared with placebo, with a between-group difference of $12.3 \mathrm{~mm}$ in favor of dienogest $(P<0.0001)$. Biberoglu and Behrman scale scores supported the visual analog scores by demonstrating greater reductions in the intensity of symptoms and signs in the dienogest group compared with placebo. The Clinical 
Global Impression scale, a measure of overall improvement, showed that $52.9 \%$ of the patients treated with dienogest were assessed by physicians to be "very much improved/much improved" at week 12, compared with $22.9 \%$ in the placebo group. Quality of life analyses based on the Short Form 36 Health Survey questionnaire, a non-disease-specific tool used widely in clinical trials, showed that dienogest was associated with significantly greater improvement in "bodily pain" than placebo treatment. Dienogest was generally well tolerated in this trial, with no serious or unexpected adverse events, and few events related to therapy. Estradiol levels were modestly suppressed, and profiles of uterine bleeding in the dienogest group were comparable with the placebo group.

Three studies of 16-24 weeks' duration have directly compared dienogest against GnRH agonists, a standard therapy in endometriosis. ${ }^{56-58}$ In a 24-week, randomized study of 252 women with endometriosis in Europe, dienogest $2 \mathrm{mg}$ once daily and leuprolide acetate at standard dose $(3.75 \mathrm{mg}$, depot intramuscular injection every four weeks) provided comparable, continuous reductions in pelvic pain measured by visual analog score. By week 24, reductions in mean visual analog score were $47.5 \mathrm{~mm}$ for dienogest and $46.0 \mathrm{~mm}$ for leuprolide acetate. Consistent with the visual analog score changes, dienogest and leuprolide acetate induced similar decreases in the intensity of symptoms and physical findings assessed by Biberoglu and Behrman scores. Short Form 36 Health Survey scores showed a trend to greater improvement in both physical and mental health in the dienogest than the leuprolide acetate group. Effects of estrogen deprivation were substantially more common in the leuprolide acetate group than dienogest group, including numbers of days with hot flushes/week (mean 4.7 versus 0.82 , respectively), while bleeding episodes were suppressed less with dienogest than leuprolide acetate. The leuprolide acetate group showed a reduction in mean lumbar bone mineral density, while, in the dienogest group, lumbar bone mineral density showed a small increase $\left(P=0.0003\right.$ versus leuprolide acetate).$^{57}$

Dienogest $1 \mathrm{mg}$ twice daily was compared with triptorelin $3.75 \mathrm{mg}$ (depot intramuscular injection every four weeks) as a postoperative medication in a 16-week, multicenter, open, randomized trial of 142 women with stage II-IV endometriosis. ${ }^{56}$ The two medications were equally effective in improving endometriosis symptoms and signs, and laparoscopy scores. Hot flushes were reported in $61.2 \%$ of women receiving the GnRH agonist compared with $9.6 \%$ of women treated with dienogest. Irregular bleeding was the most common complication in the dienogest group (61.6\% versus 25.4\% with GnRH agonist).
The third comparative trial including a GnRH agonist was a 24-week, randomized, double-blind, multicenter study comparing dienogest $2 \mathrm{mg}$ once daily against intranasal buserelin acetate $300 \mu \mathrm{g}$ three times daily, which was performed in Japan in 271 women with confirmed endometriosis. ${ }^{58}$ Dienogest was as effective as buserelin acetate in reducing endometriosis symptoms and lesion size at laparoscopy. Greatest changes in quality of life (assessed by Short Form 36 Health Survey score) in both groups were improvements in bodily pain, with a trend to greater improvement in the dienogest group. Mean lumbar bone mineral density decreased in both groups in this population, with a significantly greater reduction in the buserelin acetate group $(P=0030)$. Consistent with the other comparative trials, dienogest was associated more frequently with irregular bleeding but less frequently with hot flushes than the GnRH agonist.

Notably, each of the trials that investigated dienogest $2 \mathrm{mg}$ daily described adverse effects of generally mild to moderate intensity which were associated with low rates of treatment discontinuation (eg, $5.0 \% 0^{57}$ and $4.4 \% 0^{58}$ over 24-week treatment durations). Bleeding episodes decreased in number and intensity over time, and were associated with no discontinuations in these trials. ${ }^{57,58}$

An open-label pilot study of dienogest at the higher dose of $10 \mathrm{mg}$ twice daily for 24 weeks provides additional information on the safety of this medication. ${ }^{59,60}$ Dienogest at $20 \mathrm{mg}$ daily had no clinically relevant effect on thyroid or adrenal function, electrolyte balance, or hematopoiesis in the 21 women with stage I-IV endometriosis who participated. In addition, dienogest at this high dose had no adverse effects on lipid metabolism, liver enzymes, fasting insulin, or glucose. No menopausal symptoms and no adverse androgen-related effects were reported. These observations, demonstrating that dienogest has a favorable safety and tolerability profile even at $20 \mathrm{mg}$ daily (ie, 10 times the recommended dose), support the conclusion that the recommended dose of $2 \mathrm{mg}$ once daily will have a similar beneficial profile.

\section{Dienogest and fertility considerations}

The majority of women suffering from endometriosis are of reproductive age and so may require contraception. Based on the available data, dienogest provides complete ovulation inhibition at a daily dose of $2 \mathrm{mg} \cdot{ }^{37,42,61}$ However, dienogest monotherapy was not developed as a contraceptive, and women taking dienogest as a treatment for endometriosis are advised to use nonhormonal methods of contraception. 
Women with endometriosis may desire pregnancy once sufficient pain relief is achieved. Recent pharmacodynamic data in volunteers indicate that ovarian activity resumes rapidly (range 1-43 days) after cessation of dienogest. ${ }^{42}$ These observations support studies that describe a prompt return to fertility (eg, mean about 30 days) and include cases of successful pregnancy in women with endometriosis following the cessation of dienogest treatment $2 \mathrm{mg}$ daily for durations up to one year. ${ }^{52,62,63}$

As with other progestins, there are limited data on the use of dienogest in pregnant women. The available data do not indicate harmful effects for dienogest with respect to reproductive toxicity and reveal no special risks on pregnancy. However, dienogest should not be administered to pregnant women because there is no need to treat endometriosis during pregnancy. ${ }^{64}$

\section{Dienogest for the long-term treatment of endometriosis}

Effective management of endometriosis over the longer term is an important objective. The painful symptoms and impairment in quality of life associated with endometriosis may persist or deteriorate in the absence of effective treatment. Recurrence is frequent even after successful surgery, while there are only limited trial data to confirm the efficacy and safety of long-term treatment for many medications used in endometriosis.

Dienogest has been investigated as a long-term treatment of endometriosis in two large trials performed in Europe and Japan, which included assessments of efficacy, change in quality of life, safety, and tolerability.

Women who completed the 12-week placebo-controlled study in Europe ${ }^{54}$ were offered the opportunity to enter an open-label extension study of dienogest for up to 53 additional weeks, providing an overall treatment period of up to 65 weeks. ${ }^{62}$ Notably, of the 188 women completing the placebo-controlled study, a large proportion $(n=168$, $89 \%$ ) consented to enter the long-term extension study. The intensity of pain showed significant, sustained improvements during the long-term study, in addition to the improvements associated with dienogest during the placebo-controlled phase. Mean visual analog scores decreased from $56.9 \mathrm{~mm}$ at baseline of the placebo-controlled study to $34.1 \mathrm{~mm}$ at baseline of the long-term study, to $11.5 \mathrm{~mm}$ at the end of the 53 additional weeks of treatment (Figure 2). During a 24-week treatment-free period following the long-term study, visual analog scores increased only moderately, suggesting that dienogest induces a beneficial effect that may persist after treatment cessation. Short Form 36 Health Survey scores during the treatment-free period indicated minimal changes in physical or mental indices of quality of life over six months after cessation of dienogest.

During the long-term study, laboratory parameters, vital signs, and body weight remained stable or underwent minimal changes. Adverse effects considered potentially treatment-related developed in $16.1 \%$ of women, including breast discomfort $(4.2 \%)$, nausea $(3.0 \%)$, and irritability (2.4\%). The maximal intensity of treatment-related adverse events was mild or moderate in $92.5 \%$ of cases. In agreement with trends observed in the 12- and 24-week studies, the intensity and frequency of bleeding reduced progressively over the course of the long-term study. During post-treatment follow-up, bleeding returned to normal intensity and cyclic patterns resumed within 4-6 weeks. Treatment compliance during the long-term study was high (98\%) and discontinuation rates due to adverse events or lack of efficacy were both low ( $2.4 \%$ and $0.6 \%$, respectively).

The results of this long-term study performed in Europe are supported by a 52-week, nonrandomized trial of dienogest $2 \mathrm{mg}$ daily conducted in Japan on 135 women with confirmed endometriosis. ${ }^{63}$ Global improvement was measured by change in the severity of five subjective symptoms (lower abdominal pain, lumbago, dyschezia, dyspareunia, and pain on vaginal examination) and two objective findings (induration involving pouch of Douglas and uterine mobility). Moderate or marked global improvement was recorded in $72.5 \%$ of patients after 24 weeks and in $90.6 \%$ after 52 weeks of dienogest treatment (Figure 3). Changes in visual analog score for lower abdominal pain and lumbago decreased progressively, while the proportion of patients demonstrating a reduction in cyst size $>25 \%$ was $85 \%$ at 52 weeks. Quality of life assessments using the Short Form 36 Health Survey score indicated improvements in bodily pain by 23.57 and 27.37 points (on a 100-point scale) at 24 and 52 weeks, respectively, compared with baseline. Patient satisfaction with dienogest at the end of treatment was high, with $88.9 \%$ of women responding that they were "certainly willing" or "would prefer" to use dienogest again.

The most commonly reported treatment-related adverse event was metrorrhagia $(71.9 \%)$, followed by headaches $(18.5 \%)$ and constipation $(10.4 \%)$. None of the treatmentrelated adverse events was rated as serious. Metrorrhagia resolved in 96 of the 97 affected patients either during the study or within two months of study cessation. The frequency of bleeding lessened as treatment progressed, so that $40.5 \%$ of women were experiencing no bleeding by 

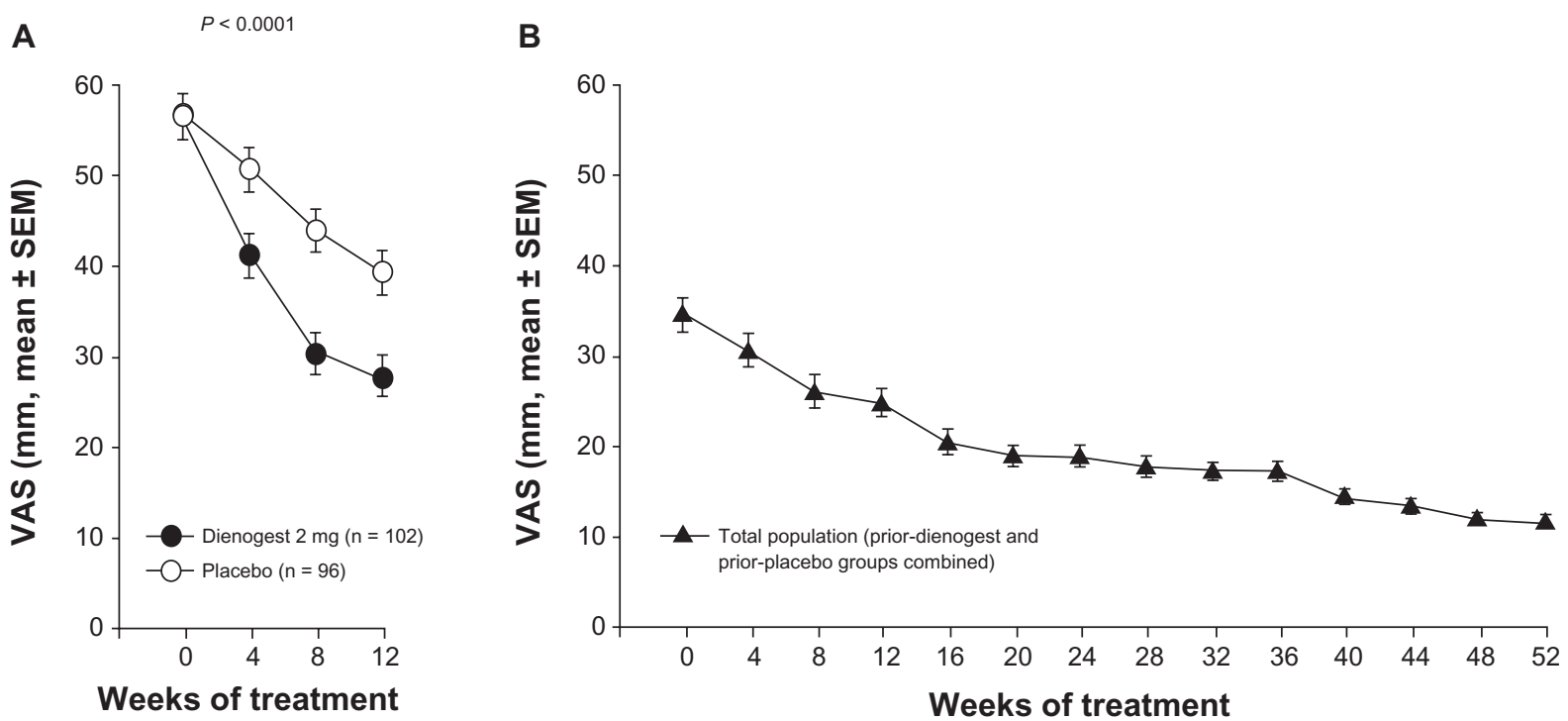

Figure 2 Change in visual analog scale (VAS) score during the placebo-controlled (A) and the extension (B) studies..$^{54,62}$ Note: Reprinted in part from Strowitzki T et al, ${ }^{54}$ with permission from Elsevier.

49-52 weeks. Resumption of menses was confirmed in all women at the end of the study. The discontinuation rate due to treatment-related adverse events was $5.2 \%$. Lumbar bone mineral density decreased by $1.7 \pm 2.2 \%$ between baseline and week 52, with the greatest change in the first 24 weeks. The authors noted that this bone mineral density change may be considered mild and not significantly greater than that observed in untreated women of similar age. No biochemical markers of bone metabolism indicated changes outside the normal reference range.

Recently, a small open-label, nonrandomized study performed in Japan has investigated continued dienogest
$2 \mathrm{mg} /$ day for 12 months ( $\mathrm{n}=33$ women) in comparison with sequential treatment including GnRH agonist (leuprorelin acetate or buserelin acetate) for 4-6 months followed by dienogest $1 \mathrm{mg}$ /day for 12 months $(\mathrm{n}=38){ }^{65}$ Continued dienogest significantly reduced the mean visual analog scores for dysmenorrhea, nonmenstrual pelvic pain, and dyspareunia at six and 12 months, equivalent to the score reductions achieved with GnRH agonist followed by dienogest. For approximately $40 \%$ of women in the sequential treatment group, the dienogest dose was increased from $1 \mathrm{mg}$ to $1.5-2 \mathrm{mg} /$ day to optimize bleeding control. Consistent with other studies, uterine bleeding was significantly reduced in

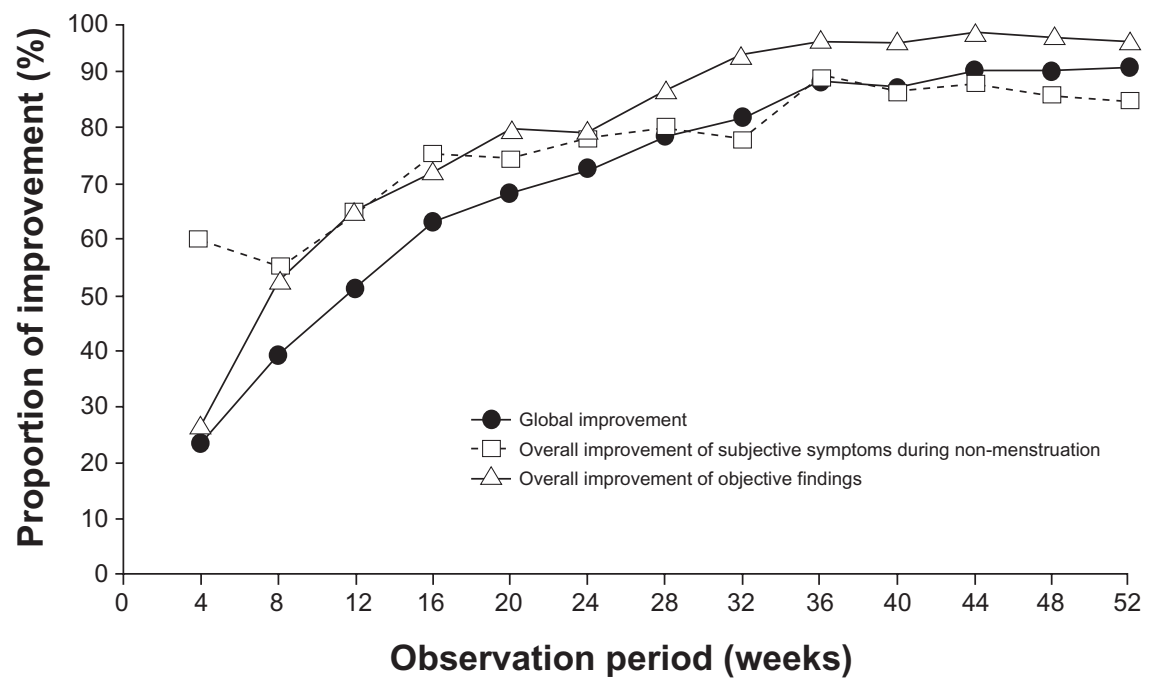

Figure 3 Increase over time in the proportion of cases assessed as "marked or moderate" for global improvement, overall improvement of subjective symptoms during non-menstruation, and overall improvement of objective findings. ${ }^{58}$

Note: Reprinted from Momoeda et $\mathrm{a}^{63}$ with permission from John Wiley \& Sons. 
the second compared with the first six months of dienogest treatment. The divergence in dienogest dose hinders the interpretation of bleeding rates across the treatment groups. The authors concluded that dienogest represents a practical and efficient long-term therapy in patients who respond to GnRH agonist therapy. The data may be interpreted also to indicate that continued long-term dienogest is as effective for pain relief as a GnRH agonist followed by dienogest therapy.

\section{Discussion}

Effective long-term management of endometriosis is a significant clinical objective in view of the debilitating nature of this often chronic condition. Among current management approaches, recurrence is common after surgery, while evidence to support the efficacy and safety of many medications is lacking. GnRH agonists, a standard medication in endometriosis, may not be administered continuously for more than six months in the absence of add-back therapy because of the deleterious hypoestrogenic effects.

Dienogest is a progestin investigated for the treatment of endometriosis. Dienogest at a dose of $2 \mathrm{mg}$ daily has been studied extensively in clinical trial programs performed in Europe and Japan, including two studies with treatment durations of up to 65 weeks. These studies demonstrated that dienogest has an efficacy, safety, and tolerability profile that is favorable for long-term use. The intensity of pain associated with endometriosis decreased progressively, adverse events (mostly mild or moderate in intensity) were predictable and associated with low discontinuation rates, and bleeding irregularities reduced in intensity and frequency over time. Following cessation of dienogest treatment, menses returned to normal.

The impact of endometriosis on a woman's quality of life is substantial and wide ranging. Quality of life assessments in the dienogest studies indicated that improvements in both physical and mental indices were attained within 12-week and 24-week treatment durations, and that these benefits were sustained in studies of up to one year.

Also of major significance to clinical practice, compliance with dienogest treatment was high and rates of discontinuation due to adverse events were low in these studies. Contributors to this favorable compliance may include the efficacy of dienogest for symptom control and the predictability of adverse effects, which can be communicated to patients before treatment initiation. Large proportions of the women who were questioned expressed a willingness to continue dienogest treatment.
Together, the dienogest study programs performed in Europe and Japan indicate that dienogest represents a promising new medication for the long-term management of endometriosis.

\section{Disclosure}

The author declares that he has no conflict of interest in this work.

\section{References}

1. Rogers PA, D'Hooghe TM, Fazleabas A, et al. Priorities for endometriosis research: Recommendations from an international consensus workshop. Reprod Sci. 2009;16(4):335-346.

2. Nasir L, Bope ET. Management of pelvic pain from dysmenorrhea or endometriosis. J Am Board Fam Pract. 2004;17 Suppl: S43-S47.

3. Eskenazi B, Warner ML. Epidemiology of endometriosis. Obstet Gynecol Clin North Am. 1997;24(2):235-258.

4. Kennedy S, Bergqvist A, Chapron C, et al. ESHRE guideline for the diagnosis and treatment of endometriosis. Hum Reprod. 2005; 20(10):2698-2704.

5. Sinaii N, Plumb K, Cotton L, et al. Differences in characteristics among 1,000 women with endometriosis based on extent of disease. Fertil Steril. 2008;89(3):538-545.

6. Holoch KJ, Lessey BA. Endometriosis and infertility. Clin Obstet Gynecol. 2010;53(2):429-438.

7. Fagervold B, Jenssen M, Hummelshoj L, Moen MH. Life after a diagnosis with endometriosis - a 15 years follow-up study. Acta Obstet Gynecol Scand. 2009;88(8):914-919.

8. Huntington A, Gilmour JA. A life shaped by pain: women and endometriosis. J Clin Nurs. 2005;14(9):1124-1132.

9. Fourquet J, Gao X, Zavala D, et al. Patients' report on how endometriosis affects health, work, and daily life. Fertil Steril. 2010;93(7): 2424-2428.

10. Kennedy S. What is important to the patient with endometriosis? Br J Clin Pract Suppl. 1991;72:8-10.

11. Jones G, Jenkinson C, Kennedy S. The impact of endometriosis upon quality of life: A qualitative analysis. J Psychosom Obstet Gynaecol. 2004;25(2):123-133.

12. Lemaire GS. More than just menstrual cramps: Symptoms and uncertainty among women with endometriosis. J Obstet Gynecol Neonatal Nurs. 2004;33(1):71-79.

13. Kjerulff KH, Erickson BA, Langenberg PW. Chronic gynecological conditions reported by US women: Findings from the National Health Interview Survey, 1984 to 1992. Am J Public Health. 1996;86(2): 195-199.

14. Denny E. Women's experience of endometriosis. $J$ Adv Nurs. 2004; 46(6):641-648.

15. Nnoaham KE, Sivananthan S, Hummelshoj L, et al. Global study of women's health: A multi-centre study of the global impact of endometriosis. Human Reproduction. 2010;25 Suppl 1:i9-i11.

16. Sepulcri RP, do Amarai VF. Depressive symptoms, anxiety, and quality of life in women with pelvic endometriosis. Eur J Obstet Gynecol Reprod Biol. 2009;142(1):53-56.

17. Gao X, Outley J, Botteman M, et al. Economic burden of endometriosis. Fertil Steril. 2006;86(6):1561-1572.

18. Winkel CA, Scialli AR. Medical and surgical therapies for pain associated with endometriosis. J Womens Health Gend Based Med. 2001; 10(2):137-162.

19. Heinrichs WL, Henzl MR. Human issues and medical economics of endometriosis. Three- vs six-month GnRH-agonist therapy. $J$ Reprod Med. 1998;43 Suppl 3:299-308. 
20. Mathias SD, Kuppermann M, Liberman RF, Lipschutz RC, Steege JF. Chronic pelvic pain: Prevalence, health-related quality of life, and economic correlates. Obstet Gynecol. 1996;87(3):321-327.

21. Mirkin D, Murphy-Barron C, Iwasaki K. Actuarial analysis of private payer administrative claims data for women with endometriosis. J Manag Care Pharm. 2007;13(3):262-272.

22. No authors listed. Revised American Society for Reproductive Medicine classification of endometriosis: 1996. Fertil Steril. 1997; 67(5):817-821.

23. The Practice Committee of the American Society for Reproductive Medicine. Treatment of pelvic pain associated with endometriosis. Fertil Steril. 2008;90 Suppl 5:S260-S269.

24. Guo SW. Recurrence of endometriosis and its control. Hum Reprod Update. 2009;15(4):441-461.

25. Allen C, Hopewell S, Prentice A, Gregory D. Nonsteroidal antiinflammatory drugs for pain in women with endometriosis. Cochrane Database Syst Rev. 2009;2:CD004753.

26. Schindler AE. Non-contraceptive benefits of hormonal contraceptives. Minerva Ginecol. 2010;62(4):319-329.

27. Davis L, Kennedy SS, Moore J, Prentice A. Modern combined oral contraceptives for pain associated with endometriosis. Cochrane Database Syst Rev. 2007;3:CD001019.

28. Vercellini P, Eskenazi B, Consonni D, et al. Oral contraceptives and risk of endometriosis: A systematic review and meta-analysis. Hum Reprod Update. 2011;17(2):159-170.

29. Brown J, Pan A, Hart RJ. Gonadotrophin-releasing hormone analogues for pain associated with endometriosis. Cochrane Database Syst Rev. 2010;12:CD008475.

30. Sagsveen M, Farmer JE, Prentice A, Breeze A. Gonadotrophin-releasing hormone analogues for endometriosis: Bone mineral density. Cochrane Database Syst Rev. 2003;4:CD001297.

31. Mounsey AL, Wilgus A, Slawson DC. Diagnosis and management of endometriosis. Am Fam Physician. 2006;74(4):594-600.

32. Selak V, Farquhar C, Prentice A, Singla A. Danazol for pelvic pain associated with endometriosis. Cochrane Database Syst Rev. 2007; 4:CD000068.

33. Muneyyirci-Delale O, Karacan M. Effect of norethindrone acetate in the treatment of symptomatic endometriosis. Int J Fertil Womens Med. 1998;43(1):24-27.

34. Telimaa S, Puolakka J, Ronnberg L, Kauppila A. Placebo-controlled comparison of danazol and high-dose medroxyprogesterone acetate in the treatment of endometriosis. Gynecol Endocrinol. 1987;1(1):13-23.

35. Lippert TH, Mueck AO. The clinical importance of dienogest. In: Teichmann AT, editor. Dienogest - Preclinical and Clinical Features of a New Progestogen. Berlin, Germany: Walter de Gruyter; 1995.

36. Mueck AO. What makes dienogest a unique progestogen for the treatment of endometriosis? Gynaecol Forum. 2010;15(2):18-23.

37. Oettel M, Breitbarth H, Elger W, et al. The pharmacological profile of dienogest. Eur J Contracept Reprod Health Care. 1999;4(S1):2-13.

38. Herkert O, Kuhl H, Sandow J, Busse R, Schini-Kerth VB. Sex steroids used in hormonal treatment increase vascular procoagulant activity by inducing thrombin receptor (PAR-1) expression: role of the glucocorticoid receptor. Circulation. 2001;104(23):2826-2831.

39. Köhler G, Faustmann TA, Gerlinger C, Seitz C, Mueck AO. A doseranging study to determine the efficacy and safety of 1,2 , and $4 \mathrm{mg}$ of dienogest daily for endometriosis. Int J Gynaecol Obstet. 2010; 108(1):21-25.

40. Sasagawa S, Shimizu Y, Kami H, et al. Dienogest is a selective progesterone receptor agonist in transactivation analysis with potent oral endometrial activity due to its efficient pharmacokinetic profile. Steroids. 2008;73(2):222-231.

41. Sasagawa S, Shimizu Y, Nagaoka T, Tokado H, Imada K, Mizuguchi K. Dienogest, a selective progestin, reduces plasma estradiol level through induction of apoptosis of granulosa cells in the ovarian dominant follicle without follicle-stimulating hormone suppression in monkeys. J Endocrinol Invest. 2008;31(7):636-641.
42. Klipping C, Duijkers I, Faustmann T, Klein SF, Schuett B. Pharmacodynamic study of four oral dosages of dienogest. Fertil Steril. 2010;94 Suppl 1:S181.

43. Katayama H, Katayama T, Uematsu K, et al. Effect of dienogest administration on angiogenesis and hemodynamics in a rat endometrial autograft model. Hum Reprod. 2010;25(11):2851-2858.

44. Harada T, Taniguchi F. Dienogest: A new therapeutic agent for the treatment of endometriosis. Womens Health (Lond Engl). 2010; $6(1): 27-35$.

45. Nakamura M, Katsuki Y, Shibutani Y, Oikawa T. Dienogest, a synthetic steroid, suppresses both embryonic and tumor-cell-induced angiogenesis. Eur J Pharmacol. 1999;386(1):33-40.

46. Okada H, Nakajima T, Yoshimura T, Yasuda K, Kanzaki H. The inhibitory effect of dienogest, a synthetic steroid, on the growth of human endometrial stromal cells in vitro. Mol Hum Reprod. 2001; 7(4):341-347.

47. Katsuki Y, Takano Y, Futamura Y, et al. Effects of dienogest, a synthetic steroid, on experimental endometriosis in rats. Eur J Endocrinol. 1998;138(2):216-226.

48. Fu L, Osuga Y, Morimoto C, et al. Dienogest inhibits BrdU uptake with G0/G1 arrest in cultured endometriotic stromal cells. Fertil Steril. 2008;89 Suppl 5:1344-1347.

49. Shimizu Y, Mita S, Takeuchi T, Notsu T, Mizuguchi K, Kyo S. Dienogest, a synthetic progestin, inhibits prostaglandin E(2) production and aromatase expression by human endometrial epithelial cells in a spheroid culture system. Steroids. 2011;76(1-2):60-67.

50. Tatsumi H, Kitawaki J, Tanaka K, Hosoda T, Honjo H. Lack of stimulatory effect of dienogest on the expression of intercellular adhesion molecule- 1 and vascular cell adhesion molecule- 1 by endothelial cell as compared with other synthetic progestins. Maturitas. 2002;42(4): 287-294.

51. Horie S, Harada T, Mitsunari M, Taniguchi F, Iwabe T, Terakawa N. Progesterone and progestational compounds attenuate tumor necrosis factor alpha-induced interleukin-8 production via nuclear factor kappa B inactivation in endometriotic stromal cells. Fertil Steril. 2005;83(5):1530-1535.

52. Momoeda M, Taketani Y. Randomized double-blind, multicentre, parallel-group dose-response study of dienogest in patients with endometriosis. Jpn Pharmacol Ther. 2007;35:769-783. Japanese.

53. Barbieri RL. Hormone treatment of endometriosis: the estrogen threshold hypothesis. Am J Obstet Gynecol. 1992;166:740-745.

54. Strowitzki T, Faustmann T, Gerlinger C, Seitz C. Dienogest in the treatment of endometriosis-associated pelvic pain: A 12-week, randomized, double-blind, placebo-controlled study. Eur J Obstet Gynecol Reprod Biol. 2010;151(2):193-198.

55. Gallagher EJ, Liebman M, Bijur PE. Prospective validation of clinically important changes in pain severity measured on a visual analog scale. Ann Emerg Med. 2001;38(6):633-638.

56. Cosson M, Querleu D, Donnez J, et al. Dienogest is as effective as triptorelin in the treatment of endometriosis after laparoscopic surgery: Results of a prospective, multicenter, randomized study. Fertil Steril. 2002;77(4):684-692.

57. Strowitzki T, Marr J, Gerlinger C, Faustmann T, Seitz C. Dienogest is as effective as leuprolide acetate in treating the painful symptoms of endometriosis: A 24-week, randomized, multicentre, open-label trial. Hum Reprod. 2010;25(3):633-641.

58. Harada T, Momoeda M, Taketani Y, et al. Dienogest is as effective as intranasal buserelin acetate for the relief of pain symptoms associated with endometriosis - a randomized, double-blind, multicenter, controlled trial. Fertil Steril. 2009;91(3):675-681.

59. Schindler AE, Christensen B, Henkel A, Oettel M, Moore C. High-dose pilot study with the novel progestogen dienogestin patients with endometriosis. Gynecol Endocrinol. 2006;22(1):9-17.

60. Schindler AE, Henkel A, Moore C, Oettel M. Effect and safety of high-dose dienogest $(20 \mathrm{mg} /$ day $)$ in the treatment of women with endometriosis. Arch Gynecol Obstet. 2010;282(5):507-514. 
61. Moore C, Carol W, Graser T, Mellinger U, Walter F. Influence of dienogest on ovulation in young fertile women. Clin Drug Invest. 1999; 18:271-278

62. Petraglia F, Hornung D, Seitz C, et al. Reduced pelvic pain in women with endometriosis: efficacy of long-term dienogest treatment. Arch Gynecol Obstet. June 17, 2011. [Epub ahead of print.]

63. Momoeda M, Harada T, Terakawa N, et al. Long-term use of dienogest for the treatment of endometriosis. J Obstet Gynaecol Res. 2009; 35(6):1069-1076.
64. Visanne ${ }^{\circledR}$. Summary of Product Characteristics. 2010. Available from: http://visanne.com/en/auth/prescribing-essentials/spc/index.php. Accessed May 25, 2011.

65. Kitawaki J, Kusuki I, Yamanaka K, Suganuma I. Maintenance therapy with dienogest following gonadotropin-releasing hormone agonist treatment for endometriosis-associated pelvic pain. Eur J Obstet Gynecol Reprod Biol. April 5, 2011. [Epub ahead of print].

\section{Publish your work in this journal}

The International Journal of Women's Health is an international, peerreviewed open-access journal publishing original research, reports, reviews and commentaries on all aspects of women's healthcare including gynecology, obstetrics, and breast cancer. Subject areas include: Chronic conditions (migraine headaches, arthritis, osteoporosis);
Endocrine and autoimmune syndromes; Sexual and reproductive health; Psychological and psychosocial conditions. The manuscript management system is completely online and includes a very quick and fair peer-review system. Visit http://www.dovepress.com/ testimonials.php to read real quotes from published authors. 